\title{
Intermittent Aerosol Delivery to the Lungs During High-Flow Nasal Cannula Therapy
}

\author{
Laleh Golshahi PhD, P Worth Longest PhD, Mandana Azimi PharmD, \\ Aamer Syed MD, and Michael Hindle PhD
}

\begin{abstract}
INTRODUCTION: Use of submicrometer particles combined with condensational growth techniques has been proposed to reduce drug losses within components of high-flow nasal cannula therapy systems and to enhance the dose reaching the lower respiratory tract. These methods have been evaluated using continuous inhalation flow rather than realistic inhalation/exhalation breathing cycles. The goal of this study was to evaluate in vitro aerosol drug delivery using condensational growth techniques during high-flow nasal cannula therapy using realistic breathing profiles and incorporating intermittent aerosol delivery techniques. METHODS: A mixer-heater combined with a vibrating mesh nebulizer was used to generate a submicrometer aerosol using a formulation of $\mathbf{0 . 2 \%}$ albuterol sulfate and $\mathbf{0 . 2 \%}$ sodium chloride in water. Delivery efficiency of the aerosol for $1 \mathrm{~min}$ through a nasal cannula was considered using an intermittent delivery regime with aerosol being emitted for either the entire inhalation time $(2 \mathrm{~s})$ or half of the inhalation period $(1 \mathrm{~s})$ and compared with continuous delivery. The deposition of the aerosol was evaluated in the nasal delivery components (ventilator tubing and cannula) and an in vitro adult nose-mouth-throat (NMT) model using 3 realistic breathing profiles. RESULTS: Significant improvements in dose delivered to the exit of the NMT model (ex-NMT) were observed for both condensational growth methods using intermittent aerosol delivery compared with continuous delivery, and increasing the tidal volume was found useful. The combination of the largest tidal volume with the shortest intermittent delivery time resulted in the lowest respiration losses and the highest ex-NMT delivered dose. CONCLUSIONS: Intermittent aerosol delivery using realistic breathing profiles of submicrometer condensational growth aerosols was found to be efficient in delivering nasally administered drugs in an in vitro airway model. Key words: high-flow nasal cannula therapy; condensational growth; submicrometer aerosol; in vitro airway model; pulmonary drug delivery; nasal cannula. [Respir Care 2014;59(10):1476-1486. ( 2014 Daedalus Enterprises]
\end{abstract}

\section{Introduction}

High-flow nasal cannula (HFNC) therapy is an increasingly common technique of oxygen delivery that allows

Drs Golshahi, Longest, Azimi, and Hindle are affiliated with the Department of Pharmaceutics, Dr Longest is affiliated with the Department of Mechanical and Nuclear Engineering, and Dr Syed is affiliated with the Division of Pulmonary Disease and Critical Care Medicine, Department of Internal Medicine, Virginia Commonwealth University, Richmond, Virginia.

The authors are employees of Virginia Commonwealth University. Virginia Commonwealth University is seeking patent applications with respect to the technology described, which if licensed may result in a financial interest to the authors. for higher flows than conventional low-flow therapy. Heating and humidification of the breathing gas allow delivery of flows of up to $45 \mathrm{~L} / \mathrm{min}$ in adults via nasal cannula. ${ }^{1}$

This study was funded by National Institutes of Health grant R01 HL10733.

Dr Hindle presented a version of this paper at the 19th International Society of Aerosols in Medicine (ISAM 2013) Congress, held April 6-10, 2013, in Chapel Hill, North Carolina.

Correspondence: Michael Hindle PhD, Department of Pharmaceutics, Room 442, Virginia Commonwealth University, 410 N 12th Street, Richmond, VA 23298-0533. E-mail: mhindle@vcu.edu.

DOI: $10.4187 /$ respcare. 02903 
The HFNC systems consist of humidification modules and HFNCs. Ward ${ }^{2}$ recently reviewed 7 observational and controlled studies assessing the efficacy of HFNC therapy and concluded that "HFNC can effectively be used to treat patients with moderate levels of hypoxemic respiratory failure." As this technique becomes more widely employed, it is appropriate to consider methods of aerosol administration through the nasal cannula that will allow efficient drug deposition in the lungs.

Previous studies have demonstrated the challenge of delivering aerosols through the nose to the lungs. These studies have focused on the evaluation of aerosol delivery at low flows using nasal cannulas. Bhashyam et $\mathrm{al}^{3}$ studied delivery of aerosol from an Aeroneb Solo mesh nebulizer (Aerogen, Galway, Ireland) through adult, pediatric, and infant cannulas at $3 \mathrm{~L} / \mathrm{min}$ inspiratory oxygen flows downstream of a heater/humidifier system. The drug output efficiency of the cannulas was $18.6-26.9 \%$ of the loaded

\section{See the Related Editorial on Page 1608}

dose in the presence of inhalation flow, which demonstrated the high drug losses within the tubing and cannula even at low flows. In a similar study, Ari et $\mathrm{al}^{4}$ studied aerosol delivery from the Aeroneb Solo device with heliox (helium-oxygen mixture) and oxygen through a pediatric nasal cannula (Optiflow, Fisher \& Paykel Healthcare, Auckland, New Zealand) at 3 and $6 \mathrm{~L} / \mathrm{min}$. The aerosol delivery efficiency at $3 \mathrm{~L} / \mathrm{min}$ was $\sim 11 \%$ with both oxygen and heliox. However, the efficiency of aerosol delivery was reduced to $\sim 5 \%$ at $6 \mathrm{~L} / \mathrm{min}$ with heliox, which was higher than the aerosol delivery using oxygen $(\sim 2 \%)$ at the same flow.

As HFNC therapy becomes more widely employed, commercial devices such as the Vapotherm aerosol adapter (Vapotherm, Stevensville, Maryland) have been designed to interface with the Aeroneb nebulizers to enable nebulizer administration during HFNC therapy. Perry et $\mathrm{al}^{5}$ studied the efficiency of albuterol delivery with a Vapotherm humidified HFNC system. The Aeroneb Solo device was used to deliver albuterol through infant, pediatric, and adult cannulas via the Vapotherm aerosol adapter downstream of a Vapotherm 2000i unit. At flows of 5, 10, 20, and $40 \mathrm{~L} / \mathrm{min}$, the delivered doses through the adult cannula were $2.5,0.8,0.4$, and $0.2 \%$, respectively. For the pediatric cannula at $3,5,10$, and $20 \mathrm{~L} / \mathrm{min}$, the inspired doses were $1.2,0.6,0.1$, and $0.0 \%$, respectively. At 3, 5, and $8 \mathrm{~L} / \mathrm{min}, \sim 0.5 \%$ of albuterol was delivered through the infant cannula. The majority $(62-80 \%)$ of albuterol was accumulated in the aerosol adapter. A combination of many factors, including high flows, humidification, small nasal prongs, and the sharp changes in the direction of the aerosol flow, resulted in the observed low efficiency of

\section{QUICK LOOK}

\section{Current knowledge}

Heated and humidified high-flow nasal cannula therapy allows for higher flows than conventional low-flow therapy. Additionally, the high flow can wash out the upper airway dead space and reduce ventilatory requirements.

\section{What this paper contributes to our knowledge}

Aerosol delivery during noninvasive ventilation is associated with significant drug losses in the delivery components and poor lung deposition. In a model system, controlled condensational growth aerosols and intermittent delivery via high-flow nasal cannula were associated with $>70 \%$ delivery of the emitted dose.

in vitro aerosol delivery during HFNC therapy. Poor lung delivery efficiency can be expected as a result of high drug depositional losses in the device components and the extrathoracic airways. , $^{, 6-8}$

High losses in the ventilation components and the extrathoracic airways could be avoided by decreasing the size of the aerosol to reduce impaction losses, which are inherent at high flows, and consequently deliver improved drug doses to the lungs. With this goal in mind, Longest et $\mathrm{al}^{9}$ proposed the use of separate streams of submicrometer aerosol and heated humidified air to the left and right nostrils, respectively, of a physically realistic in vitro nosemouth-throat (NMT) model. The submicrometer aerosol, generated by evaporating the output of the small-particle aerosol generator, had low deposition in the delivery device and was shown to efficiently penetrate the nasal model and subsequently increase to micrometer size when mixed with the heated and humidified airstream beyond the nasal septum. This co-administration of heated humidified air, as used in HFNC therapy, causes the enhanced condensational growth (ECG) of the submicrometer aerosol to micrometer size and was hypothesized to enable lung deposition and prevent aerosol exhalation. Longest et $\mathrm{al}^{9}$ were successful in decreasing the drug depositional losses in the NMT model to $15 \%$ using a submicrometer aerosol compared with $73 \%$ losses in the NMT model with a $4.7-\mu \mathrm{m}$ mass median aerodynamic diameter aerosol generated from a conventional nebulizer. Limitations of this initial proofof-concept study include the failure to employ a realistic patient cannula and the method of aerosol generation, which was the less commonly used small-particle aerosol generator. ${ }^{9}$

Hindle and Longest ${ }^{10}$ proposed a second approach using the same controlled condensational growth principle to deliver an inhaled submicrometer aerosol in combination with a hygroscopic excipient. The submicrometer aerosol 
has minimal depositional losses in the extrathoracic airways. However, in this case, due to the use of combination drug and hygroscopic excipient particles, when the aerosol is exposed to the natural humidity of the respiratory system, excipient-enhanced growth (EEG) occurs, producing micrometer-sized droplets suitable for deposition. This technique has been evaluated for use with on-demand spray inhalers such as the Respimat ${ }^{11}$ and with newly developed powder inhalers. ${ }^{12}$

Golshahi et al ${ }^{13}$ described the development and initial in vitro testing of an aerosol delivery setup to enable both ECG and EEG methods during HFNC therapy. Steadystate studies were performed using a constant inhalation flow to assess device deposition, NMT model deposition, and aerosol growth. The aerosol delivery setup consisted of a low-volume vibrating mesh nebulizer (Aeroneb Lab) and a mixer-heater ${ }^{14}$ to produce the submicrometer aerosol. The mixer-heater uses heated compressed gas (37$43^{\circ} \mathrm{C}$ ) to evaporate the aerosol droplet output of the commercial nebulizer, reducing its mass median aerodynamic diameter from 4-6 $\mu \mathrm{m}$ to $<1 \mu \mathrm{m}$. The development of the mixer-heater was described previously by Longest et al ${ }^{14}$ and characterized as having a high emitted dose $(>90 \%)$ and aerosol mass median aerodynamic diameter of 0.91 (0.08) $\mu \mathrm{m}$ when tested under steady-state flow conditions. ${ }^{14}$ Aerosols were delivered to the in vitro nasal model using 2 nasal cannulas ${ }^{7,14}$ designed for the ECG and EEG methods. The nasal cannulas were designed using a streamlining approach to minimize aerosol drug deposition by avoiding sharp edges, which may cause rapid change in the direction of aerosol stream and consequently impaction losses. ${ }^{7}$ Golshahi et al ${ }^{13}$ observed that, under steady inhalation flow conditions (without an exhalation cycle), for a $0.2 \%$ albuterol sulfate and $0.2 \%$ sodium chloride nebulized formulation, the mean drug doses delivered through the nasal cannula and the in vitro NMT model were $88.5 \%$ and $78 \%$ of the nominal dose using the EEG and ECG growth protocols, respectively.

In addition, the particle size of the original submicrometer-sized particles when measured following passage through the NMT at the trachea was $1.55 \mu \mathrm{m}$ for EEG delivery and $2.82 \mu \mathrm{m}$ for ECG delivery. Golshahi et al ${ }^{13}$ demonstrated the feasibility of delivering a submicrometer aerosol during HFNC therapy with minimal deposition in both the delivery device and the nasal passages. However, this study was performed using steady-state inhalation flow without an exhalation cycle. It is to be expected that delivery efficiency would be decreased in the presence of an exhalation flow, which during cyclical breathing would prevent the aerosol entering the in vitro NMT model. It is already well established that aerosol delivery during the exhalation cycle creates a high exhaled fraction, which is a significant challenge for effective lung deposition of aerosols administered to invasively ventilated patients. ${ }^{15}$
Among the strategies employed to minimize exhalation losses, intermittent aerosol drug delivery has been used for numerous applications such as intermittent positive-pressure ventilation and drug delivery to the sinuses. ${ }^{16-19}$ Using intermittent aerosol delivery, aerosol is delivered only during the inhalation portion of the breathing cycle to minimize exhalation losses. However, the effectiveness of this method has been controversial and has not been obvious in all applications. ${ }^{20}$ In this study, we extend the previous work of Golshahi et $\mathrm{al}^{13}$ and incorporate realistic breathing cycles into the in vitro testing to compare continuous aerosol drug delivery using controlled condensational growth methods (ECG and EEG) with 2 modes of intermittent aerosol delivery compared with continuous aerosol delivery.

\section{Methods}

\section{Aerosol Mixer-Heater System and In Vitro Nasal Delivery Setup}

An aerosol delivery system was developed to generate heated submicrometer aerosols for delivery to subjects receiving HFNC therapy. To initiate aerosol delivery, the ventilation gas flow was diverted to the mixer-heater into which the nebulized aerosol was generated. For EEG delivery, the ventilation gas was supplied at a flow of $20 \mathrm{~L} / \mathrm{min}$ directly to the mixer-heater to deliver the submicrometer aerosol as a single-flow stream via a cannula. For ECG delivery, ventilation gas $(20 \mathrm{~L} / \mathrm{min})$ was again supplied to the mixer-heater, and a separate stream of HFNC therapy flow $\left(15 \mathrm{~L} / \mathrm{min}, 43^{\circ} \mathrm{C}\right.$, relative humidity $\left.>95 \%\right)$ was delivered to the gas inlet of a divided cannula. The components of the aerosol mixer-heater system and in vitro test setup are shown schematically in Figure 1A and have been described previously ${ }^{13}$ with the following modifications. First, a breathing simulator (ASL 5000, Ingmar Medical, Pittsburgh, Pennsylvania) was incorporated into the in vitro model to produce realistic breathing profiles during aerosol generation. The breathing profiles employed are described in Table 1 and are characterized by a sinusoidal waveform with peak inspiratory flows of 23,35 , and $44 \mathrm{~L} / \mathrm{min}$ for profiles $1-3$, respectively, while maintaining a breathing frequency of 15 breaths/min. Second, compressed air was supplied to the mixer-heater at a flow of $20 \mathrm{~L} / \mathrm{min}$ using 3 protocols (continuous flow, $2 \mathrm{~s}$ on and $2 \mathrm{~s}$ off, and $1 \mathrm{~s}$ on and $3 \mathrm{~s}$ off) to enable continuous and intermittent aerosol delivery, respectively. This was achieved using a 3-way solenoid valve controlled by a timer and was synchronized such that, during intermittent delivery, the air was delivered during the 2-s inhalation cycle, as shown in Figure 2. The compressed air was heated as it entered the mixer-heater by Kapton heaters embedded 


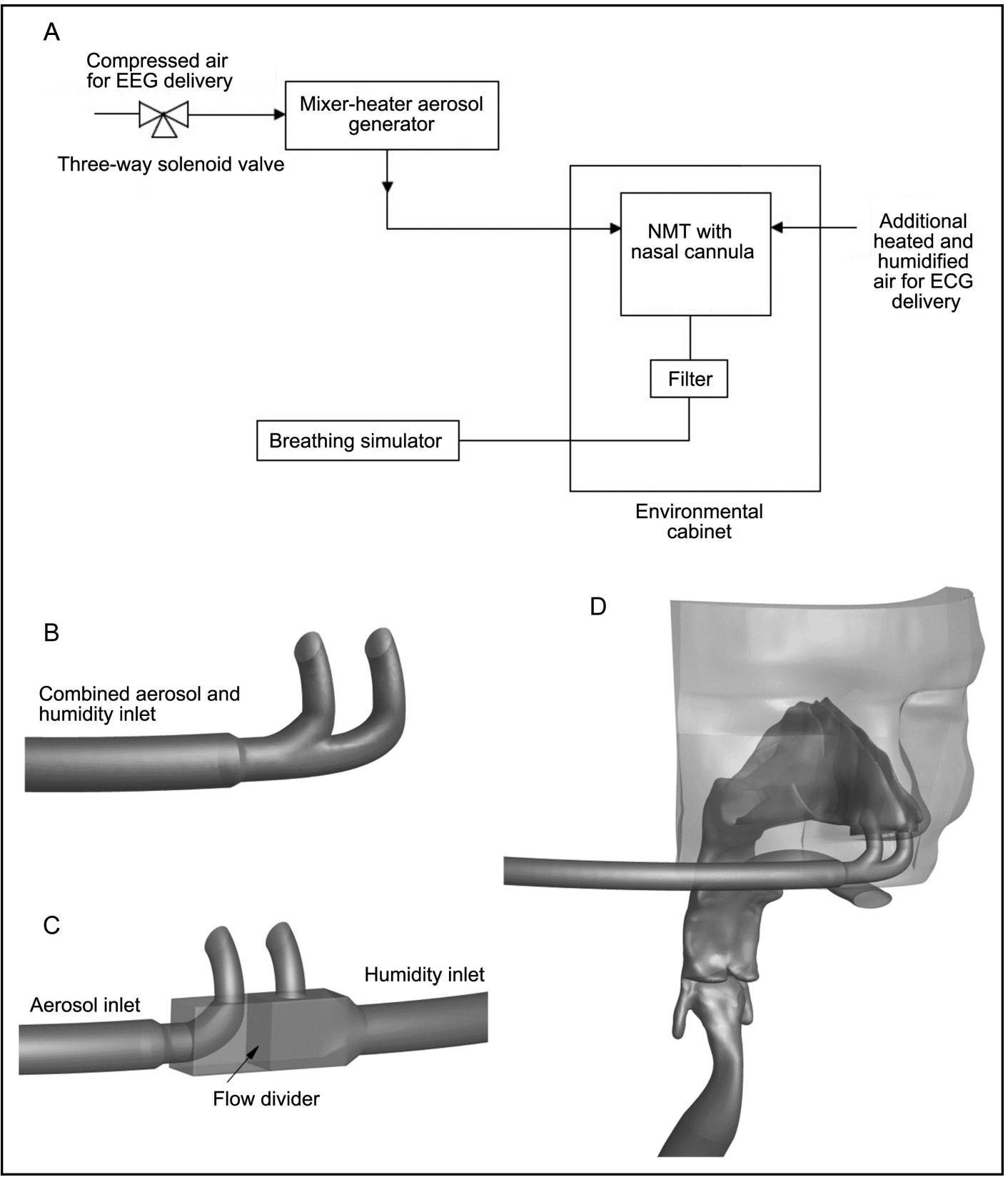

Fig. 1. The experimental setup for in vitro controlled condensational growth. A: Schematic diagram. B: Excipient-enhanced growth (EEG) nasal cannula. C: Enhanced condensational growth (ECG) nasal cannula. D: Nose-mouth-throat (NMT) model, including the facial structure, shown with EEG nasal cannula.

in its walls (Omegalux KHLV-202, Omega Engineering, Stamford, Connecticut).

The remainder of the setup consisted of the aerosol mixer-heater, ${ }^{14}$ which was used to generate the submicrometer aerosol by mixing the droplets emitted from a vibrating mesh nebulizer (Aeroneb Lab) with the heated compressed air stream. The nebulizer formulation contained $0.2 \%$ albuterol sulfate and $0.2 \%$ sodium chloride in deionized water. The duration of the nebulization time and aerosol delivery was $1 \mathrm{~min}$, which was the equivalent of
15 breathing cycles at a frequency of 15 breaths/min (see Table 1). It should be noted that the nebulization and delivery time could be adjusted according to the required delivered dose. For these studies, the time was optimized to provide sufficient drug recovery to enable analytical detection by high-performance liquid chromatography. Ventilator tubing (70-cm length and $10-\mathrm{mm}$ internal diameter) was used to deliver the aerosol from the mixerheater to the nasal cannula interface. For EEG delivery, an EEG streamlined single inlet cannula ${ }^{7}$ was employed, and 
Table 1. Simulated Breathing Profiles Measured at the Nostrils of the Nose-Mouth-Throat Model

\begin{tabular}{lcccc}
\hline \hline $\begin{array}{c}\text { Breathing } \\
\text { Profile }\end{array}$ & $\begin{array}{c}\text { Peak } \\
\text { Inspiratory } \\
\text { Flow (L/min) }\end{array}$ & $\begin{array}{c}\text { Inhaled } \\
\text { Volume, } \\
\mathrm{V}_{\mathrm{T}}(\mathrm{L})\end{array}$ & $\begin{array}{c}\text { Breathing } \\
\text { Frequency } \\
\text { (Breaths/min) }\end{array}$ & $\begin{array}{c}\text { Average } \\
\text { Inhalation } \\
\text { Flow (L/min)* }\end{array}$ \\
\hline 1 & 23 & 0.50 & 15 & 15 \\
2 & 35 & 0.75 & 15 & 22.5 \\
3 & 44 & 0.93 & 15 & 27.9
\end{tabular}

$* 2 \times \mathrm{V}_{\mathrm{T}} \times \mathrm{f}$, where $\mathrm{V}_{\mathrm{T}}$ is tidal volume, and $\mathrm{f}$ is breathing frequency

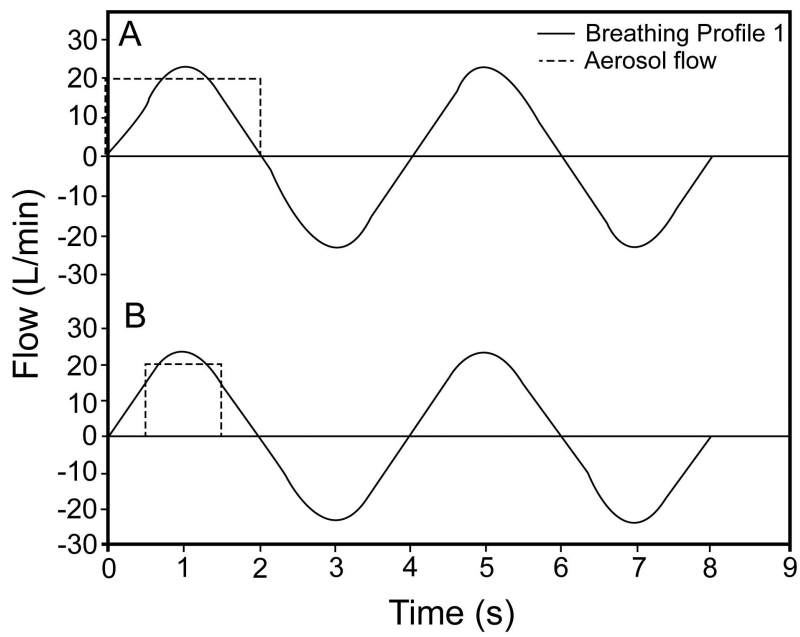

Fig. 2. A: Intermittent 2-s aerosol delivery. B: Intermittent 1-s aerosol delivery during simulation of breathing profile 1 .

the aerosol flow from the mixer-heater was delivered via the cannula (see Fig. 1B) to the NMT model at a flow of $20 \mathrm{~L} / \mathrm{min}$. For ECG delivery, a divided ECG cannula, ${ }^{14}$ with separate inlets for the aerosol and heated humidified high-flow air, was employed. The aerosol was delivered at $20 \mathrm{~L} / \mathrm{min}$ from the mixer-heater to the aerosol side of the cannula, and the additional humidified high-flow air at a flow of $15 \mathrm{~L} / \mathrm{min}$ and a temperature of $43^{\circ} \mathrm{C}$ was delivered to the humidity side (see Fig. 1C). This high flow air was generated using a Vapotherm 2000i unit. The cannulas were positioned at the nostrils of a physically realistic NMT model, ${ }^{9}$ as shown in Figure 1D. The temperature of the aerosol exiting the cannula was $37 \pm 2^{\circ} \mathrm{C}$. At the exit of the NMT model, a low-resistance filter (PulmoGuard II, Quest Diagnostics, Brockton, Massachusetts) was employed to collect drug penetrating the model and was connected to the breathing simulator as shown in Figure 1A. The NMT model was placed in an environmental chamber (Espec, Hudsonville, Michigan) at $37 \pm 0.5^{\circ} \mathrm{C}$ with relative humidity $>90 \%$.

The details of the NMT model have been described previously. ${ }^{9,13}$ In brief, the NMT model included the face for positioning the nasal cannulas in the nostrils, nasal cavity (including turbinates), nasopharynx, a portion of the oral cavity, pharynx, larynx, and trachea (see Fig. 1D). Since all of these anatomic features were not available in a single high-quality computed tomography scan, several scans of healthy adult males with an age range of 35-54 y were selected to develop the characteristic model. The nasal airway of this characteristic model is based on the frequently used magnetic resonance imaging dataset of Guilmette et al, ${ }^{21}$ which is based on the images taken from an adult male ( $40 \mathrm{y}$ old).

\section{Emitted Dose and In Vitro Deposition Measurement and Analysis}

To quantify the dose passing through to the nasal delivery components during a 1-min period of aerosolization, a PulmoGuard II filter was positioned at the outlet of the mixer-heater, with the rest of the in vitro components downstream of this filter. The mass of drug collected on this filter using breathing profile 1 was determined as the mixer-heater emitted dose.

For both EEG and ECG delivery modes, the effect of continuous and intermittent delivery ( 2 and $1 \mathrm{~s}$ ) on device deposition and aerosol delivery efficiency was investigated using 3 breathing patterns (profiles 1-3). However, comparison of continuous and intermittent delivery was done using only breathing profile 1 as a single control case because we hypothesized that the amount of loss due to delivery during exhalation in continuous mode would be the same using the 2 other profiles. Ex-NMT delivered dose was defined as the percentage of emitted dose deposited on the filter at the exit of the NMT model and was used as an in vitro estimate of the dose delivered to the lungs. Following aerosol generation and delivery, the nasal delivery setup components were detached and rinsed using known volumes of deionized water. The mass of albuterol sulfate on each component was determined using a validated high-performance liquid chromatography analytical method ${ }^{22}$ and used to calculate the percentage of drug deposition (expressed as a percentage of the emitted dose).

\section{Statistical Analysis}

Each experiment was repeated 3-4 times. One-way analysis of variance, followed by the Tukey honest significant difference post hoc test, was performed to compare continuous delivery with the 2 intermittent delivery modes. Two-way analysis of variance, followed by the 2-tailed Student $t$ test and Tukey honest significant difference test, was used to study the effect of timing mode (1-s vs 2-s modes) and breathing pattern (profiles 1-3), respectively. All statistical tests were performed using JMP Pro 10.0.2 
(JMP, Cary, North Carolina), with a $P$ value of $<.05$ as the indicator of significance.

\section{Results}

\section{Aerosol Emitted Dose From the Mixer-Heater}

The albuterol sulfate dose (mean \pm SD) emitted from the mixer-heater during continuous air delivery was $82.4 \pm 2.2 \%$ of the nominal dose using breathing profile 1. The emitted doses (mean $\pm \mathrm{SD}$ ) during 2-s and 1-s intermittent deliveries were $73.6 \pm 2.5 \%$ and $76.3 \pm 5.9 \%$ of the nominal dose using breathing profile 1 , respectively. The nominal dose was determined based on the weight difference of the nebulizer before and after nebulization and the measured albuterol sulfate concentration of the formulation. The emitted dose during continuous air delivery was significantly different from the intermittent delivery modes $(P=.02)$. The emitted dose during continuous delivery was significantly higher than that during 2 -s intermittent delivery $(P=.02)$, but there was no significant difference in emitted dose between continuous and 1 -s deliveries $(P=.12)$. There was no significant difference in the emitted dose between 2-s and 1-s deliveries $(P=.62)$.

\section{Comparison of Continuous and Intermittent EEG and ECG Aerosol Deliveries}

The amount of drug collected on the PulmoGuard II filter at the outlet of the NMT model was denoted as the ex-NMT delivered dose and can be considered as an estimate of the dose delivered to the lungs. Figure 3 shows this amount as a percentage of the emitted dose for ECG and EEG protocols. The mode of delivery (continuous, 1-s, or 2-s) had a significant effect on lung delivery in both protocols (ECG: $P<.001$; EEG: $P<.001$ ) using breathing profile 1 . Lung delivery was significantly higher with intermittent air delivery compared with continuous air delivery in both protocols using breathing profile $1(P<.001)$.

A portion of drug was released to the environmental cabinet through the spaces between the cannula and the nostrils during respiration. This amount is called respiration loss and was estimated by subtracting the total amount of the drug recovered from all the deposition sites downstream of the mixer-heater from the emitted dose. Figure 4 shows these calculated respiration losses for ECG and EEG protocols, respectively. The mode of delivery (continuous, 1-s, or 2-s) had a significant effect on respiration losses in both ECG and EEG protocols using breathing profile 1 (ECG: $P<.001$; EEG: $P<.001)$. Respiration losses were significantly lower during both 1 -s and 2-s intermittent air deliveries compared with continuous air delivery using breathing profile $1(P<.001)$.

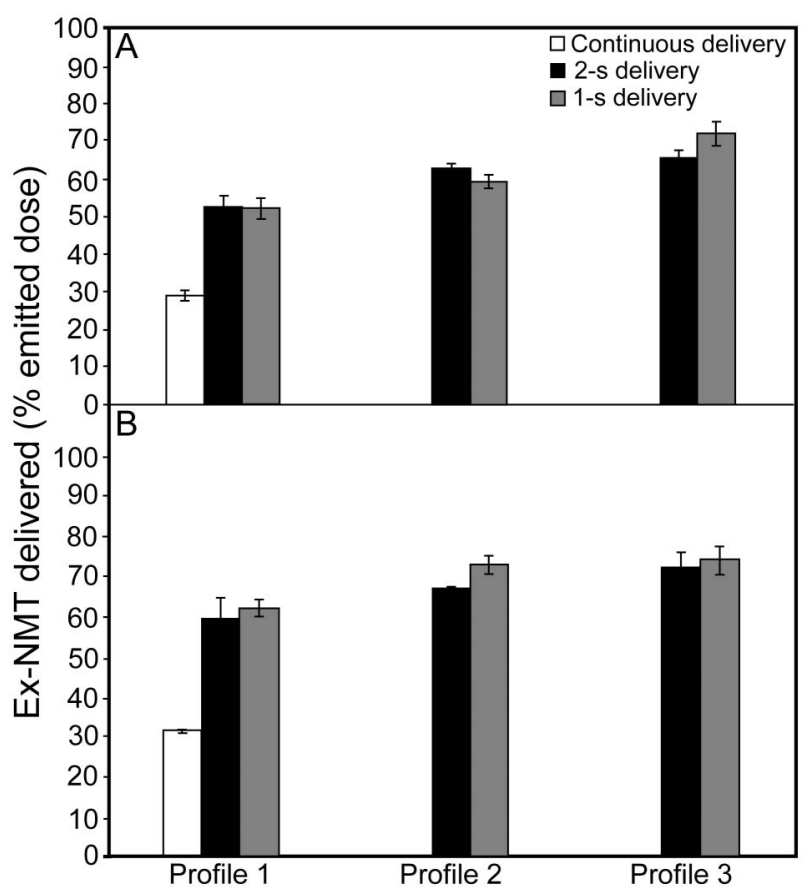

Fig. 3. Amount of drug captured at the outlet (ex-NMT) of the nose-mouth-throat (NMT) model. A: Delivered dose as a percentage of the emitted dose in the enhanced condensation growth (ECG) delivery modes and B: excipient enhanced growth (EEG) delivery modes. Data are shown as mean \pm SD.

Drug depositions in the device components as percentages of the emitted dose are given in Tables 2 and 3 for ECG and EEG protocols using breathing profile 1, respectively. Overall deposition in these components was low $(<10.6 \%)$, indicating the efficiency of the ECG and EEG delivery methods. Device losses were significantly higher with 1-s delivery compared with continuous delivery in both ECG $(P=.001)$ and EEG $(P=.007)$ protocols. Overall device deposition with 2-s delivery was significantly higher than continuous delivery in EEG mode $(P=.02)$.

Deposition in the NMT model is given in Tables 4 and 5 for the ECG and EEG protocols, respectively, and was reasonably low in all cases $(<6.6 \%)$, demonstrating the delivery of the submicrometer aerosols through the nasal model. Mode of delivery had a significant effect on nasal losses with both protocols (ECG: $P<.001$;EEG: $P=.036$ ). Nasal losses during 1-s delivery with ECG $(P<.001)$ and 2-s delivery with EEG $(P=.03)$ were significantly higher than that during continuous delivery.

\section{Effect of Breathing Profile on EEG and ECG Aerosol Deliveries}

The breathing profile showed a significant effect on lung delivery for both EEG $(P<.001)$ and ECG $(P<.001)$ 


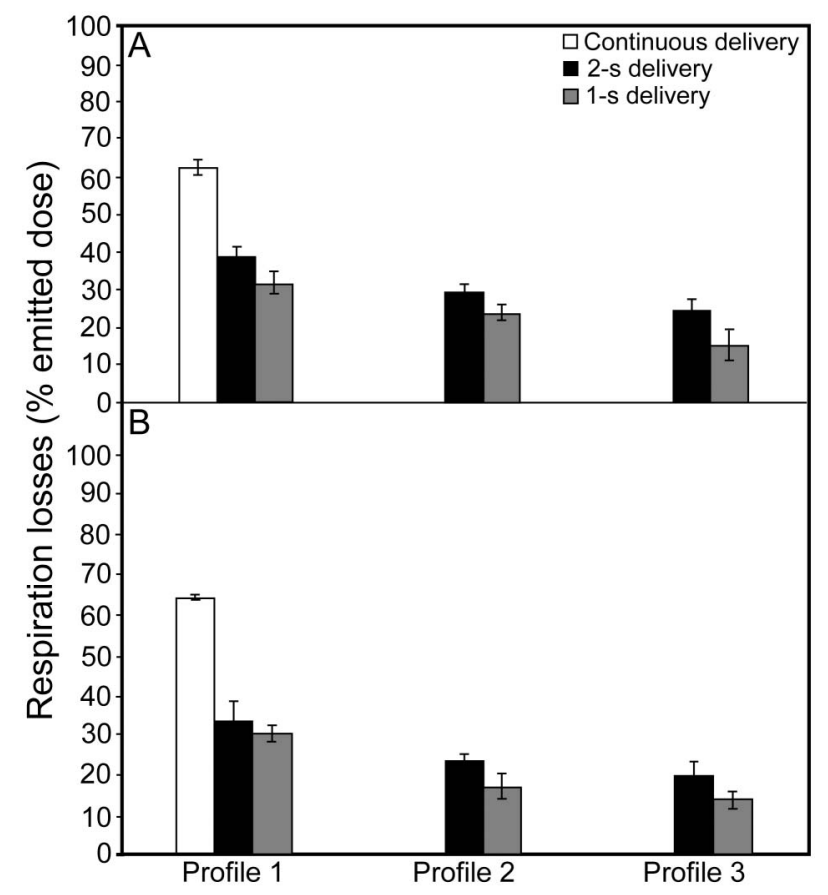

Fig. 4. A: Respiration losses as a percentage of the emitted dose in all enhanced condensation growth (ECG) delivery modes and B: all excipient enhanced growth (EEG) delivery modes. Data are shown as mean \pm SD.

protocols (see Fig. 3). However, there was a significant interaction between the breathing profile and the duration of intermittent delivery for the ex-NMT delivered dose with ECG $(P=.004)$, suggesting that the effect of breathing was not the same for each of the 2 intermittent delivery modes (1-s and 2-s) with ECG. For ECG, increasing the tidal volume $\left(\mathrm{V}_{\mathrm{T}}\right)$ from 0.75 to $0.93 \mathrm{~L}$ significantly increased the ex-NMT delivered dose from $59.1 \pm 2.1 \%$ to $72.0 \pm 3.2 \%$ using the 1 -s delivery mode $(P<.001)$, but there was no difference using the 2 -s mode $(P=.55)$. For both 1-s and 2-s delivery modes, increasing the $\mathrm{V}_{\mathrm{T}}$ from 0.5 to $0.75 \mathrm{~L}$ significantly increased the ex-NMT delivered dose using ECG delivery. No significant interaction was found between breathing profile and duration of intermittent delivery for EEG $(P=.51)$, and increasing the $\mathrm{V}_{\mathrm{T}}$ from 0.5 to $0.75 \mathrm{~L}$ and $0.93 \mathrm{~L}$ significantly increased the ex-NMT delivered dose $(P<.001)$.

The breathing profile also showed a significant effect on respiration losses for both EEG $(P<.001)$ and ECG $(P<.001)$ (see Fig. 4). Increasing the $\mathrm{V}_{\mathrm{T}}$ from 0.5 to $0.75 \mathrm{~L}$ significantly decreased the respiration losses for both EEG $(P<.001)$ and ECG $(P<.001)$. Increasing the $\mathrm{V}_{\mathrm{T}}$ from 0.75 to $0.93 \mathrm{~L}$ also significantly decreased the respiration losses for ECG $(P=.001)$, but with EEG, the losses with these tidal volumes were not significantly different.
Breathing profiles showed no significant effect on the device losses for both ECG $(P=.07)$ and EEG $(P=.21)$ (see Tables 2 and 3). Breathing profiles did not have a significant effect on the NMT deposition $(P=.36)$ for ECG, but showed a significant effect for EEG $(P<.001)$ (see Tables 4 and 5). There was also a significant interaction between the breathing pattern and the mode of delivery (1-s and 2-s) with EEG $(P=.001)$, which indicates that the effect of breathing on nasal deposition is different for each of the intermittent delivery modes (1-s and 2-s). During 1-s delivery, increasing the $\mathrm{V}_{\mathrm{T}}$ from 0.5 to $0.75 \mathrm{~L}$ $(P=.02)$ and $0.93 \mathrm{~L}(P<.001)$ significantly increased the deposition in the NMT model. However, during 2-s delivery, the deposition in the NMT model with all breathing profiles was not significantly different $(P>.05)$.

\section{Effect of Duration of Intermittent Delivery on EEG and ECG Aerosol Performance}

The duration of intermittent delivery did not show a significant effect on ex-NMT delivered dose with ECG $(P=.48)$, but showed a significant effect with EEG $(P=.02)$ (see Fig. 3). However, with ECG, due to significant interaction between breathing and the time duration of intermittent delivery, the effect of duration should be considered for each breathing pattern individually. This comparison showed that using a shorter delivery time increased the ex-NMT delivered dose significantly $(P=.03)$ with the maximum $\mathrm{V}_{\mathrm{T}}(0.93 \mathrm{~L}$, profile 3$)$, but the difference between the 2 modes (1-s and 2-s) in ex-NMT delivered dose with breathing profiles $1(P>.99)$ and 2 $(P=.42)$ was not significant. With EEG, reducing the time of intermittent delivery (1-s vs 2-s) consistently increased the ex-NMT delivered dose for all 3 breathing patterns $(P=.02)$.

The duration of the intermittent delivery showed a significant effect on respiration losses for both ECG $(P<.001)$ and EEG $(P=.001)$ (see Fig. 4). Using 1-s instead of 2-s delivery significantly reduced the respiration losses with both ECG $(P<.001)$ and EEG $(P=.001)$ protocols.

The duration of delivery showed a significant effect on total device losses for ECG $(P<.001)$, but no significant effect for EEG $(P=.54)$ (see Tables 2 and 3). Reducing the duration of intermittent delivery significantly increased the total device losses in ECG $(P<.001)$. The effect of duration of intermittent delivery on deposition in the NMT model was significant for both ECG $(P=.001)$ and EEG $(P=.001)$ protocols (see Tables 4 and 5). For ECG, decreasing the time duration significantly increased the losses in the NMT model $(P=.001)$. However, considering the significant interaction between the breathing and the time duration $(P=.001)$ for EEG, the 2 timing modes should be compared for each breathing profile separately. This comparison showed that the losses in the NMT model 


\section{InTERmitTent Aerosol Delivery During HFNC Therapy}

Table 2. Deposition as a Percentage of the Emitted Dose in Each Device Component During Continuous and Intermittent Enhanced Condensation Growth Delivery Modes and Simulation of Three Breathing Profiles

\begin{tabular}{|c|c|c|c|c|c|c|c|}
\hline \multirow[b]{2}{*}{ Delivery Mode } & \multicolumn{3}{|c|}{$\begin{array}{l}\text { Breathing Profile } 1 \\
\quad(\text { Mean } \pm \mathrm{SD})\end{array}$} & \multicolumn{2}{|c|}{$\begin{array}{c}\text { Breathing Profile } 2 \\
(\text { Mean } \pm \mathrm{SD})\end{array}$} & \multicolumn{2}{|c|}{$\begin{array}{c}\text { Breathing Profile } 3 \\
(\text { Mean } \pm \mathrm{SD})\end{array}$} \\
\hline & Continuous & $2-\mathrm{s}$ & $1-\mathrm{s}$ & $2-s$ & $1-\mathrm{s}$ & $2-s$ & $1-\mathrm{s}$ \\
\hline Nebulizer & $0.9 \pm 0.4$ & $0.5 \pm 0.1$ & $0.9 \pm 0.2$ & $1.1 \pm 0.6$ & $0.9 \pm 0.3$ & $0.9 \pm 0.4$ & $0.4 \pm 0.1$ \\
\hline Tubing* & $3.2 \pm 0.6$ & $4.6 \pm 0.6 \dagger$ & $5.5 \pm 0.7 \dagger$ & $3.1 \pm 1.0$ & $5.9 \pm 1.0$ & $3.5 \pm 1.2$ & $4.3 \pm 1.2$ \\
\hline Cannula $*+$ & $1.4 \pm 0.4$ & $1.0 \pm 0.3$ & $4.1 \pm 1.4 \dagger \S$ & $1.3 \pm 0.3$ & $3.8 \pm 1.4 \S$ & $1.1 \pm 0.1$ & $2.6 \pm 0.9 \S$ \\
\hline Total Device** $*$ & $5.5 \pm 0.7$ & $6.1 \pm 0.4$ & $10.5 \pm 2.1 \dagger \S$ & $5.5 \pm 0.4$ & $10.6 \pm 2.3 \S$ & $5.5 \pm 1.5$ & $7.3 \pm 2.1 \S$ \\
\hline \multicolumn{8}{|c|}{$\begin{array}{l}* P<.05 \text {, significant effect of delivery mode (ie, intermittent vs continuous) using profile } 1 \text { (one-way analysis of variance) } \\
\dagger P<.05 \text {, significant difference compared with continuous delivery (Tukey honest significant difference post hoc) } \\
\ddagger P<.05 \text {, significant effect of timing of intermittent delivery (no significant effect of breathing profile; no significant interaction between timing and breathing modes [2-way analysis of variance]) } \\
\S P<.05 \text {, significant difference between } 2 \text {-s and 1-s deliveries (post hoc } 2 \text {-tailed Student } t \text { test) }\end{array}$} \\
\hline
\end{tabular}

Table 3. Deposition as a Percentage of the Emitted Dose in Each Device Component During Continuous and Intermittent Excipient-Enhanced Growth Delivery Modes and Simulation of Three Breathing Profiles

\begin{tabular}{|c|c|c|c|c|c|c|c|}
\hline \multirow[b]{2}{*}{ Delivery Mode } & \multicolumn{3}{|c|}{$\begin{array}{l}\text { Breathing Profile } 1 \\
\quad(\text { Mean } \pm \mathrm{SD})\end{array}$} & \multicolumn{2}{|c|}{$\begin{array}{l}\text { Breathing Profile } 2 \\
\quad(\text { Mean } \pm \text { SD) }\end{array}$} & \multicolumn{2}{|c|}{$\begin{array}{l}\text { Breathing Profile } 3 \\
\quad(\text { Mean } \pm \mathrm{SD})\end{array}$} \\
\hline & Continuous & $2-s$ & $1-\mathrm{s}$ & $2-\mathrm{s}$ & $1-\mathrm{s}$ & $2-\mathrm{s}$ & $1-\mathrm{s}$ \\
\hline Nebulizer & $0.6 \pm 0.4$ & $0.7 \pm 0.4$ & $1.1 \pm 1.3$ & $1.2 \pm 0.4$ & $1.5 \pm 2.2$ & $0.6 \pm 0.2$ & $0.6 \pm 0.2$ \\
\hline Tubing* & $2.6 \pm 0.2$ & $4.4 \pm 1.2 \dagger$ & $4.8 \pm 0.9 \dagger$ & $6.2 \pm 0.9$ & $5.1 \pm 1.3$ & $5.3 \pm 2.1$ & $6.1 \pm 1.8$ \\
\hline Cannula & $0.2 \pm 0.2$ & $0.6 \pm 0.3$ & $0.5 \pm 0.5$ & $0.6 \pm 0.2$ & $1.2 \pm 0.8$ & $0.4 \pm 0.4$ & $0.8 \pm 0.5$ \\
\hline Total Device* & $3.4 \pm 0.4$ & $5.8 \pm 1.5 \dagger$ & $6.3 \pm 0.8 \dagger$ & $8.0 \pm 1.0$ & $7.8 \pm 3.9$ & $6.4 \pm 2.0$ & $7.6 \pm 1.9$ \\
\hline
\end{tabular}

Table 4. Deposition as a Percentage of the Emitted Dose in the Nose-Mouth-Throat Model During Different Enhanced Condensation Growth Delivery Modes

\begin{tabular}{lccc}
\hline \hline Breathing Profile & $\begin{array}{c}\text { Continuous } \\
(\text { Mean } \pm \text { SD })\end{array}$ & $\begin{array}{c}2-\mathrm{s} \\
(\text { Mean } \pm \text { SD) }\end{array}$ & $\begin{array}{c}1-\mathrm{s} \\
(\text { Mean } \pm \mathrm{SD})\end{array}$ \\
\hline Profile $1^{*}$ & $2.7 \pm 0.2$ & $2.6 \pm 0.3$ & $5.9 \pm 0.9 \dagger$ \\
Profile 2* & NA & $3.0 \pm 0.4$ & $6.6 \pm 1.4 \dagger$ \\
Profile 3* & NA & $4.4 \pm 1.6$ & $6.2 \pm 2.6 \dagger$
\end{tabular}

*P<.05, significant effect of timing of the intermittent delivery (no significant effect of breathing profile; no significant interaction between timing and breathing modes [2-way analysis of variance])

$\dagger P<.05$, significant difference between 2-s and 1-s deliveries (post hoc 2-tailed student $t$ - test)

$\mathrm{NA}=$ not applicable

were significantly higher using the 1 -s mode with profile $3(P<.001)$, but the difference between the 2 modes was not significant with profile $1(P=.98)$ and profile 2 $(P=.32)$.

\section{Discussion}

Aerosol delivery during invasive and noninvasive ventilation is known to be associated with high drug losses in
Table 5. Deposition as a Percentage of the Emitted Dose in the Nose-Mouth-Throat Model During Different ExcipientEnhanced Growth Delivery Modes

\begin{tabular}{lccc}
\hline \hline Breathing Profile & $\begin{array}{c}\text { Continuous } \\
(\text { Mean } \pm \text { SD) }\end{array}$ & $\begin{array}{c}2 \text {-s } \\
(\text { Mean } \pm \text { SD })\end{array}$ & $\begin{array}{c}1-\mathrm{s} \\
(\text { Mean } \pm \mathrm{SD})^{*}\end{array}$ \\
\hline Profile 1 & $0.3 \pm 0.2$ & $0.9 \pm 0.2$ & $0.6 \pm 0.4$ \\
Profile 2 & NA & $1.2 \pm 0.2$ & $2.1 \pm 1.1 \dagger$ \\
Profile 3† & NA & $1.4 \pm 0.2$ & $3.9 \pm 0.5 \dagger \S$
\end{tabular}

*P<.05, significant effect of timing of the intermittent delivery (2-way analysis of variance) $\dagger P<.05$, significant difference between 2- and 1-s deliveries (post hoc 2-tailed Student $t$ test)

$\ddagger P<.05$, significant effect of breathing profile (2-way analysis of variance)

$\S P<.05$, significant difference compared with profile 1 (post hoc Tukey honest significant difference)

$\mathrm{NA}=$ not applicable

the delivery components and consequently very low lung deliveries of drug, on the order of $1-10 \%$ in vitro ${ }^{23}$ and $1-6 \%$ in vivo, ${ }^{24}$ among adults. Similar low-delivery efficiencies have been reported during low-flow oxygen therapy ${ }^{3,4}$ and more recently with HFNC therapy. ${ }^{5}$ The use of submicrometer particles in combination with hygroscopic growth has previously shown positive effects on lowering device losses from $50-70 \%$ of the nominal dose, using 
conventional micrometer-sized particles, to $<20 \%$ of the dose. ${ }^{13}$ In this study, the overall losses in the device components (see Tables 2 and 3) were found to be low $(<10.6 \%)$. Total device component losses were significantly lower with continuous delivery compared with 1-s delivery (in ECG and EEG) and 2-s delivery (in EEG). It is hypothesized that a more concentrated aerosol bolus is delivered during the intermittent delivery mode compared with continuous delivery, which may increase particle impaction at these flows. An increase in aerosol concentration is expected during intermittent delivery as aerosol is continuously generated in the mixer-heater; however, aerosol emptying takes place only during 1-s and 2-s deliveries. Total device component losses were not significantly different for 1-s and 2-s deliveries using the 3 breathing profiles for the EEG delivery mode. However, for ECG, there was a significant increase in total device losses for each of the 3 breathing profiles with 1-s delivery. This may be associated with backflow of heated and humidified air through the aerosol delivery line during ECG delivery, which may cause hygroscopic growth that leads to higher impaction losses.

Depositional loss of the drug in the mixer-heater was $\sim 25 \%$ (ie, emitted dose of $75 \%$ ) during intermittent delivery, which was only slightly higher than the loss of $\sim 18 \%$ (ie, emitted dose of $82 \%$ ) during continuous delivery. This low deposition in the mixer-heater despite the intermittent delivery could be due to the relatively large reservoir size $(\sim 3 \mathrm{~L})$ and the associated low probability of sedimentation over the short intermittent period (2-3 s). Decreasing the device deposition has been shown to increase lung delivery to $74-88 \%$ of the nominal dose using a constant inspiratory flow. ${ }^{13}$ However, lower lung deliveries were expected using realistic breathing profiles based on the observed overestimation during previous in vitro studies compared with in vivo studies..$^{13}$ Lower lung delivery with realistic breathing profiles compared with steady-state inhalation was confirmed in this study, where ex-NMT delivered doses in the range of $50-75 \%$ of the dose emitted from the mixer-heater (see Fig. 3) were observed. However, this still represents a significant improvement compared with conventionally sized aerosols delivered during HFNC therapy $(<10 \%) .{ }^{5}$ Lung delivery was significantly higher with intermittent air delivery compared with continuous air delivery in both EEG and ECG protocols, suggesting success of the intermittent delivery mode due to reduced respiratory losses (see Fig. 4). Increasing the $\mathrm{V}_{\mathrm{T}}$ from 0.5 to $0.75 \mathrm{~L}$ resulted in a significant increase in the ex-NMT delivered dose with both intermittent delivery modes (1-s and 2-s) and EEG/ECG protocols due to the greater possibility of reaching the filter at the trachea with the higher $\mathrm{V}_{\mathrm{T}}$ prior to exhalation. However, further increasing the $\mathrm{V}_{\mathrm{T}}$ from 0.75 to $0.93 \mathrm{~L}$ increased the exNMT delivered dose using only 1-s ECG delivery.
The difference in the ex-NMT delivered doses using realistic breathing profiles was attributed to differences in respiration losses, which were estimated in Figure 4. Respiration losses during the intermittent delivery were significantly lower than those during the continuous delivery mode using both EEG and ECG protocols. Increasing the $\mathrm{V}_{\mathrm{T}}$ from 0.5 to $0.75 \mathrm{~L}$ resulted in lower respiration losses for both ECG and EEG protocols. There was an insignificant difference in the respiratory losses when the $\mathrm{V}_{\mathrm{T}}$ was increased from 0.75 to $0.93 \mathrm{~L}$ for the EEG mode, whereas for the ECG mode, increasing the $\mathrm{V}_{\mathrm{T}}$ consistently resulted in lower respiration losses. Moreover, the respiration losses during 1-s delivery were significantly lower compared with those during 2-s delivery in all cases. This was expected by considering Figure 2B, which shows that using 1-s delivery resulted in complete overlap of the inspiratory flow profile and the aerosol delivery flow from the mixerheater. In contrast, for the 2-s delivery (see Fig. 2A), the aerosol delivery flow exceeded the inspiratory flow for portions of the cycle, and this aerosol would likely be lost to the environment through the nostrils.

Overall drug deposition losses in the NMT model were low, with deposition being generally greater following 1-s delivery compared with the 2-s and continuous delivery modes. As hypothesized for total device component losses, the higher NMT deposition with 1-s delivery is likely due to the delivery of higher aerosol concentrations during the high-flow periods of the waveform, resulting in increased impaction.

Figure 3 shows a similar range of lung deliveries for the ECG and EEG modes (ex-NMT delivered doses in the range of 59.7-74.5\% with EEG and 52.6-72.0\% using ECG) using the intermittent delivery protocols. It was noted that total deposition in device components and the NMT model was found to be higher in the ECG mode compared with the EEG mode, which could be due to the additional constant flow in the ECG mode, which increases the probability of impaction. Respiration losses were also relatively higher in the ECG mode compared with the EEG mode, which similarly could be due to the additional compressed air delivered to the second nostril. Both methods offer unique advantages, with EEG relying upon the natural humidity of the airways, which could be considered a simpler delivery approach. In comparison, ECG offers higher aerosol growth potential due to the presence of the supersaturated high-flow nasal therapy delivered to the other nostril.

\section{Clinical Implications}

This study describes further development of an aerosol drug delivery system for use during HFNC therapy, incorporating intrinsic respiratory mechanics into in vitro testing with a model drug (albuterol sulfate). It was demon- 
strated that it was possible to improve the in vitro delivery efficiency of aerosols through nasal cannulas compared with what has been reported previously in the literature ${ }^{5}$ by using a combination of submicrometer-sized particles and controlled condensational growth. This method may allow simultaneous aerosol delivery without significant interruption of HFNC therapy. The technique employs a conventional mesh nebulizer, coupled with a simple mixer-heater, ${ }^{14}$ to produce submicrometer aerosols. This base technology would be readily applicable to other nebulized drugs required for patients with asthma exacerbations, pneumonia with severe hypoxemia, sickle cell disease (acute chest syndrome), pulmonary hypertension, cystic fibrosis, and other chronic lung infections like Mycobacterium avium complex. For these conditions, aerosol administration of 3 classes of drugs is envisioned to be more efficacious using this technology: (1) bronchodilators (for asthma, COPD exacerbations, pneumonia with severe bronchitis, congestive heart failure, etc); (2) pulmonary vasodilators (for sickle cell disease, pulmonary hypertension, embolism, and ARDS); and, of particular interest, (3) antibiotics (for cystic fibrosis or M. avium complex), which require high doses or frequent administration and could be delivered to a patient while continuing to receive HFNC therapy. This approach may also be of benefit for drugs that are prohibitively expensive such as iloprost; improved delivery efficiency to prevent drug waste may offer cost efficiency advantages. The example of albuterol sulfate demonstrated the potential benefit in terms of delivered dose and delivery time. In this study, from a nominal dose of $800 \mu \mathrm{g}$, the ex-NMT delivered dose of albuterol sulfate was 300$420 \mu \mathrm{g}$, which was achieved following $1 \mathrm{~min}$ of nebulization. For comparison, the nominal dose of a conventional mesh nebulizer is $2.5 \mathrm{mg}$, which is nebulized over a 10-15-min delivery period to achieve a delivered dose, ${ }^{25,26}$ similar to that found in the current study.

In practice, controlled condensational growth techniques could readily be implemented in current HFNC therapy systems. In the EEG mode, the oxygen therapy gas source would be diverted to the mixer-heater to deliver the submicrometer aerosol as a single-flow stream via a cannula. In this study, a flow of $20 \mathrm{~L} / \mathrm{min}$ was employed; however, with suitable validation, other flows could be employed to deliver the aerosol in a heated $\left(37^{\circ} \mathrm{C}\right)$ and humidified $(40-$ $50 \%$ ) oxygen therapy gas stream suitable for short-duration high-efficiency delivery while allowing continued respiratory support. For ECG delivery, oxygen $(20 \mathrm{~L} / \mathrm{min})$ is again supplied to the mixer-heater, and a separate stream of HFNC therapy $(15 \mathrm{~L} / \mathrm{min})$ is delivered to the gas inlet of a divided cannula. Using this mode, the patient continues to receive HFNC therapy through one nostril while aerosol is delivered to the other nostril for enhanced condensational growth. Automation of the aerosol delivery during inhalation could be achieved by using a breath- actuated solenoid valve upstream of the mixer-heater. Most current ventilators employ such breath-actuated valves for aerosol delivery during inhalation.

\section{Limitations and Future Research}

From a clinical perspective, there are some limitations in implementing this proof-of-concept study. One of the limitations of this study was positioning the filter at the trachea to estimate lung dose, which is different from in vivo conditions, where the respiratory tract extends through the conducting airways to the alveoli. Drug depositing on the filter is not available for exhalation, and this potentially leads to an overestimation of the lung dose. The patency of the nasal airways should also be considered. The in vitro model assumes that both nasal passages are patent, which may result in overestimation of the lung dose. Although it should be noted that the use of high flow in HFNC therapy tends to clear the nasal passages, the presence of nasal congestion from sinusitis or a deviated septum may limit the practical success of this approach for aerosol drug delivery, and this needs further clinical assessment. Another limitation of this study was the increased drug retention within the mixer-heater (emitted dose of 74-76\%) using intermittent delivery compared with continuous delivery. Thus, our future goals are increasing these emitted doses to higher values ( $>90 \%$ ) by improving the design of the mixer-heater, optimization for intermittent delivery, and incorporating a system for automating intermittent delivery. In addition, one important aspect of the controlled condensational growth is the manipulation of the size of the aerosol to deliver submicrometer-sized aerosol to the nasal airways and to increase their size after bypassing the nasal airways to enhance deposition in the upper tracheobronchial region or the lung alveoli, depending on the target of interest. Considering that such size measurements require a different experimental setup, determination of the rate of growth using various breathing profiles and hygroscopic excipients will be the focus of an independent study in the future.

\section{Conclusions}

The controlled growth techniques, which were previously found to be useful for steady-flow patterns, ${ }^{13}$ were tested using realistic breathing. The intermittent delivery methods were found useful in increasing the ex-NMT delivered dose significantly compared with continuous air delivery due to reducing the respiration losses approximately by half. Two modes of intermittent delivery (2-s and 1-s) were tested, and they both resulted in acceptable emitted doses (74-76\%) from the mixer-heater. However, the 1-s mode naturally resulted in lower respiration losses and higher ex-NMT delivered doses. $\mathrm{V}_{\mathrm{T}}$ had a positive 


\section{Intermittent Aerosol Delivery During HFNC Therapy}

effect on enhancing the dose; thus, the combination of intermittent delivery with deep inhalation is suggested for efficient HFNC aerosol therapy.

\section{ACKNOWLEDGMENTS}

We thank Ross Walenga and Geng Tian PhD of Virginia Commonwealth University, Richmond, Virginia, for their part in developing the mixerheater system and NMT model.

\section{REFERENCES}

1. Dysart K, Miller TL, Wolfson MR, Shaffer TH. Research in high flow therapy: mechanisms of action. Respir Med 2009;103(10):14001405.

2. Ward JJ. High-flow oxygen administration by nasal cannula for adult and perinatal patients. Respir Care 2013;58(1):98-122.

3. Bhashyam AR, Wolf MT, Marcinkowski AL, Saville A, Thomas K, Carcillo JA, Corcoran TE. Aerosol delivery through nasal cannulas: an in vitro study. J Aerosol Med Pulm Drug Deliv 2008;21(2):181188.

4. Ari A, Harwood R, Sheard M, Dailey P, Fink JB. In vitro comparison of heliox and oxygen in aerosol delivery using pediatric high flow nasal cannula. Pediatr Pulmonol 2011;46(8):795-801.

5. Perry S, Kesser K, Geller D, Selhorst D, Rendle J, Hertzog J. Influences of cannula size and flow rate on aerosol drug delivery through the Vapotherm humidified high-flow nasal cannula system. Pediatr Crit Care Med 2013;14(5):250-256.

6. Rubin BK. Pediatric aerosol therapy: new devices and new drugs. Respir Care 2011;56(9):1411-1421; discussion 1421-1423.

7. Longest PW, Golshahi L, Hindle M. Improving pharmaceutical aerosol delivery during noninvasive ventilation: effects of streamlined components. Ann Biomed Eng 2013;41(6):1217-1232.

8. Dhand R. Aerosol therapy in patients receiving noninvasive positive pressure ventilation. J Aerosol Med Pulm Drug Deliv 2012;25(2): 63-78.

9. Longest PW, Tian G, Hindle M. Improving the lung delivery of nasally administered aerosols during noninvasive ventilation: an application of enhanced condensational growth (ECG). J Aerosol Med Pulm Drug Deliv 2011;24(2):103-118.

10. Hindle M, Longest PW. Condensational growth of combination drugexcipient submicrometer particles for targeted high-efficiency pulmonary delivery: evaluation of formulation and delivery device. J Pharm Pharmacol 2012;64(9):1254-1263.

11. Longest PW, Tian G, Li X, Son YJ, Hindle M. Performance of combination drug and hygroscopic excipient submicrometer particles from a softmist inhaler in a characteristic model of the airways. Ann Biomed Eng 2012;40(12):2596-2610.
12. Son YJ, Longest PW, Tian G, Hindle M. Evaluation and modification of commercial dry powder inhalers for the aerosolization of a submicrometer excipient enhanced growth (EEG) formulation. Eur J Pharm Sci 2013;49(3):390-399.

13. Golshahi L, Tian G, Azimi M, Son YJ, Walenga R, Longest PW, Hindle M. The use of condensational growth methods for efficient drug delivery to the lungs during noninvasive ventilation high flow therapy. Pharm Res 2013;30(11):2917-2930.

14. Longest PW, Walenga RL, Son YJ, Hindle M. High efficiency generation and delivery of aerosols through nasal cannula during noninvasive ventilation. J Aerosol Med Pulm Drug Deliv 2013;26(5): 266-279.

15. Ari A, Restrepo RD. Aerosol delivery device selection for spontaneously breathing patients 2012. Respir Care 2012;57(4):613-626.

16. Möller W, Schuschnig U, Khadem Saba G, Meyer G, Junge-Hülsing B, Keller M, Häussinger K. Pulsating aerosols for drug delivery to the sinuses in healthy volunteers. Otolaryngol Head Neck Surg 2010; 142(3):382-388.

17. Dolovich MB, Killian D, Wolff RK, Obminski G, Newhouse MT. Pulmonary aerosol deposition in chronic bronchitis: intermittent positive pressure breathing versus quiet breathing. Am Rev Respir Dis 1977;115(3):397-402.

18. Camargo CA Jr, Spooner CH, Rowe BH. Continuous versus intermittent beta-agonists in the treatment of acute asthma. Cochrane Database Syst Rev 2003;(4):CD001115.

19. Bisceglia M, Belcastro A, Poerio V, Raimondi F, Mesuraca L, Crugliano C, Corapi UP. A comparison of nasal intermittent versus continuous positive pressure delivery for the treatment of moderate respiratory syndrome in preterm infants. Minerva Pediatr 2007;59(2): 91-95.

20. Mehta S, Hill NS. Noninvasive ventilation. Am J Respir Crit Care Med 2001;163(2):540-577.

21. Guilmette RA, Wicks JD, Wolff RK. Morphometry of human nasal airways using magnetic resonance imaging. J Aerosol Med 1989; 2(4):365-377.

22. Delvadia RR, Longest PW, Hindle M, Byron PR. In vitro tests for aerosol deposition. III. Effect of inhaler insertion angel on aerosol deposition. J Aerosol Med Pulm Drug Deliv 2013;26(3):145-156.

23. Hess DR. The mask for noninvasive ventilation: principles of design and effects on aerosol delivery. J Aerosol Med 2007;20(Suppl 1): S85-S98.

24. Parkes SN, Bersten AD. Aerosol kinetics and bronchodilator efficacy during continuous positive airway pressure delivered by face mask. Thorax 1997;52(2):171-175.

25. Dhand R. Inhalation therapy in invasive and noninvasive mechanical ventilation. Curr Opin Crit Care 2007;13(1):27-38.

26. Duarte AG, Momii K, Bidani A. Bronchodilator therapy with metered-dose inhaler and spacer versus nebulizer in mechanically-ventilated patients: comparison of magnitude and duration of response. Respir Care 2000;45(7):817-823.

This article is approved for Continuing Respiratory Care Education credit. For information and to obtain your CRCE

(free to AARC members) visit www.rcjournal.com 